\title{
Response discrepancy versus attitude similarity-dissimilarity as determinants of attraction'
}

\author{
DONN BYRNE, 2 STANFORD UNIVERSITY, GERALD L. CLORE, JR., UNIVERSITY OF ILLINOIS \\ WILLIAM GRIFFITT, UNIVERSITY OF TEXAS
}

In a previous investigation Nelson found that with proportion of similar attitudes experimentally controlled, subjectstranger response discrepancy has a significant effect on attraction. He hypothesized that if discrepancy were held constant, attraction would not vary as a function of similaritydissimilarity. With new data involving $31 \mathrm{Ss}$ and old data based on $120 \mathrm{Ss}$, proportion of similar attitudes significantly influenced attraction even though response discrepancy was controlled. It appears that attraction is a joint function of these two partially independent stimulus dimensions.

In attraction research the definition of "similar" and "dissimilar" attitudes held by the $S$ and the target person usually involves responses falling on the same or on the opposite side of the attitudinal neutral point. For example, if a six-point item contains three points representing varying strengths of pro-Republican and three points of pro-Democratic sentiment, similarity could refer to precisely the same response or to a response as much as two scale points distant. Dissimilarity could refer to a response from one to five scale points away.

In his doctoral research, Nelson (1965) investigated the possible effects of such response discrepancy on interpersonal attraction as distinct from the effects of similarity-dissimilarity. He utilized a 12-item attitude scale with six-point items. Each of 80 Ss responded to a bogus stranger who expressed similar responses on either all 12 items or on none of the items. Within each condition, there was either relatively small or relatively large response discrepancy. Analysis of variance indicated that each independent variable significantly influenced attraction toward the stranger; similarity elicits a more positive response than dissimilarity while a small discrepancy elicits a more positive response than a large discrepancy. Nelson speculated that similaritydissimilarity is simply a special case of discrepancy and that if discrepancy were held constant, attraction would not vary as a function of similarity-dissimilarity. The present research was designed to test Nelson's hypothesis.

Method

Each of 31 undergraduates was given a 56-item attitude scale and later presented with the scale of a bogus stranger who expressed similar responses on either 19 items (.34 similarity) or on all 56 items
(1.00 similarity). The responses of the stranger were prepared in such a way that the mean discrepancy per item was maintained between 1.43 and 1.95. The mean discrepancy of the two experimental groups was almost perfectly matched (1.64 per item in the 1.00 similarity condition and $1.63 \mathrm{per}$ item in the .34 condition). Ss were given the standard instructions concerning an interpersonal judgment task, and the strangers were evaluated on the Interpersonal Judgment Scale which contains two items that are summed to measure attraction (Byrne \& Nelson, 1965).

Results

If the Nelson hypothesis were correct, the two groups would not yield different attraction responses. If, however, the similarity variable influences attraction over and above the discrepancy effect, the groups would yield differential attraction responses. The correctness of the latter proposition is shown by the obtained attraction scores of 5.21 for the .34 group and 9.24 for the 1.00 group $(t=4.94, d f=29$, $\mathrm{p}<.001$ ).

\section{Discussion}

In an effort to obtain additional evidence, a similar analysis was carried out on previously gathered data (Byrne \& Clore, 1966) which involved the responses of 120 Ss who had evaluated a stranger on the basis of 12 attitude items. Subject-stranger discrepancy was determined, and the sample was divided into three relatively homogeneous groups with respect to discrepancy. Within each subgroup, a statistically significant $F$ ratio was found, indicating the effect of similarity-dissimilarity on attraction with discrepancy held constant.

Thus, Nelson's demonstration of the effect of discrepancy on attraction is an indication of the operation of a stimulus variable in addition to similarity-dissimilarity rather than one which is an alternative to similarity. It is suggested, then, that attraction is a joint function of these two partially independent stimulus dimensions.

To explore the joint-function possibility, data from two previous investigations (Byrne \& Clore, 1966; Byrne \& Griffitt, 1966) were reanalyzed by means of a correlational approach. Each $\mathrm{S}$ was considered with respect to proportion of similar attitudes, subjectstranger discrepancy, and the S's attraction response. With six subgroups, the two stimulus variables and 
Table 1. Attraction (1) as a Joint Function of Similarity-Dissimilarity (2) and Discrepancy (3)

\begin{tabular}{lllll} 
& $r_{12}$ & $r_{13}$ & $r_{23}$ & $r 1.23$ \\
\hline Group 1, N = 40 & .59 & -.55 & -.77 & .61 \\
Group 2, N=40 & .46 & -.34 & -.75 & .45 \\
Group 3, N = 40 & .46 & -.26 & -.73 & .47 \\
Group 4, N =95 & .23 & -.23 & -.56 & .34 \\
Group 5, N = 80 & .20 & -.37 & -.62 & .38 \\
Group 6, N =97 & .42 & -.53 & -.84 & .54
\end{tabular}

Note: Groups 1-3 are the mimeograph, tape and movie conditions described in Byrne and Clore (1966). Groups 4-6 are the 4th-6th graders, 7th-9th graders, and 10th-12th graders described in Byrne and Griffitt (1966).

the response variable were intercorrelated and a multiple correlation coefficient determined between attraction and the two independent variables. The findings are reasonably consistent across the six groups as shown in Table 1. Attraction is a positive function of similarity-dissimilarity, a negative function of discrepancy, and the two stimulus variables are substantially though not perfectly related. The six multiple correlation coefficients indicate a very small though consistent gain in predictive accuracy through the use of the joint function.

It would seem that individuals are responding to extremely small degrees of discrepancy between themselves and strangers as well as to the more gross variable of agreement versus disagreement. Not only is attraction influenced by similarity of positive or negative orientation toward a given topic but also by the specific degree of positiveness or negativeness.

\section{References}

Byme, D., \& Clore, G. L., Jr. Predicting interpersonal attraction toward strangers presented in three different stimulus modes. Psychon. Sci., 1966, 4, 239-240.

Byme, D., \& Griffitt, W. A developmental investigation of the law of attraction. J. Pers, soc. Psychol., 1966, 4, 699-702.

Byme, D., \& Nelson, D. Attraction as a linear function of proportion of positive reinforcements. J. Pers. soc. Psychol., 1965, 1, 659-663.

Nelson, D. A. The effect of differential magnitude of reinforcement on interpersonal attraction. Unpublished doctoral dissertation, University of Texas, 1965.

\section{Notes}

1. This research was supported in part by Research Grant MH11178-02 from the National Institute of Mental Health, United States Public Health Service.

2. On leave 1966-1967 from the University of Texas. 